consequences for quantum-state preparation, quantum computation and quantum complexity theory.

One way of performing quantum computation is by encoding the solution of a computational problem into the ground state of a Hamiltonian. The desired state is then obtained by approaching its Hamiltonian adiabatically from a simple initial one whose ground state is easily prepared. Typically, one would like the interactions involved to be two-body, geometrically local and requiring control of only a few local degrees of freedom (say two, as in a qubit). Proof that this is indeed possible - for example on a two-dimensional square lattice - was one of the first successes of the perturbationtheory gadgets ${ }^{2,3}$.

A related result concerns the complexity of determining the groundstate energy of a quantum system. It is known that, for classical systems, obtaining a minimum-energy configuration of a spin glass with nearest-neighbour interactions on a square lattice is the hardest problem in a notorious complexity class called NP. This class encompasses all problems whose solutions can be efficiently verified, but not necessarily found, on a classical computer. The quantum analogue of this result was derived by again making use of the aforementioned gadgets: determining the ground-state energy of pair-wise interacting qubits on a square lattice is as hard as such a problem can get; seemingly more complicated interactions or geometries do not yield harder problems ${ }^{2,3}$. In a similar vein, the complexity of widespread methods such as Hartree-Fock or density functional theory can be pinpointed ${ }^{6}$.

Usually, perturbation theory comes with a catch: the perturbative term has to be sufficiently small. In the context of the work on many-body systems, in which low-energy properties must be preserved, 'small' is defined with respect to the energy of excitations. Extensivity of the perturbations' energy thus seems to require that the energy of the lowest excitations (the spectral gap) grows linearly with the system size. Although unphysical, this is what was assumed in the earlier reductions. Bravyi et al. ${ }^{1}$ have now shown that this shortcoming can be circumvented: the coupling strength can remain independent of the system size. Nevertheless, many-body interactions can be replaced by pair-wise interactions such that the relative difference in the ground-state energies remains arbitrarily small (here the local reduction is independent of the rest of the system). The question of whether approximating the ground-state energy up to such a relative error has the same computational complexity is the basis of one of the major conjectures of quantum complexity theory - the 'quantum PCP theorem'.

Whether or not these methods shed new light on complexity, the acquired tools and gadgets certainly have the potential to be of use in many corners of theoretical physics where reductions are necessary and perturbations ubiquitous. And where gadgets don't work, there may be other widgets and gizmos to play with.

\footnotetext{
References

1. Bravyi, S., DiVincenzo, D. P., Loss, D. \& Terhal, B. M. Phys. Rev Lett. 101, 070503 (2008).

2. Kempe, J., Kitaev, A. \& Regev, O. SIAM J. Comput. 35 1070-1097 (2006).

3. Oliveira, R. I. \& Terhal, B. M. Preprint at $<$ http://arxiv.org/abs/ quant-ph/0504050> (2005).

4. Jordan, S. \& Farhi, E. Phys. Rev. A 77, 062329 (2008)

5. Biamonte, J. D. \& Love, P. J. Phys. Rev. A 78, 012352 (2008).

6. Schuch, N. \& Verstraete, F. Preprint at <http://arxiv.org/ abs/0712.0483> (2007).
}

\title{
PARTICLEPHYSICS
}

\section{Don't discount the tachyon!}

It makes regular appearances in science fiction - from the novels of Isaac Asimov, to TV's Dr Who and Babylon 5 - but the tachyon is less popular in science fact.

This hypothetical particle travels faster than light and the square of its rest mass is negative. So far, so unphysical. However, the proper treatment of tachyonic fields within quantum field theory does not allow any violation of causality, and writing in Physical Review D, John Ellis and co-authors make a plea to theorists to resist "unreasoning tachyophobia" (Phys. Rev. D 78, 075006; 2008). It could be that tachyons feature in an extended standard model.

The standard model is not the whole story for particle physics, and it is hoped that data from the Large Hadron Collider will direct us to the physics needed to extend, even if not complete, the picture. Supersymmetry - a broken symmetry that would endow each fundamental particle with a heavier supersymmetric partner - is a favoured addition to the model, typically in the

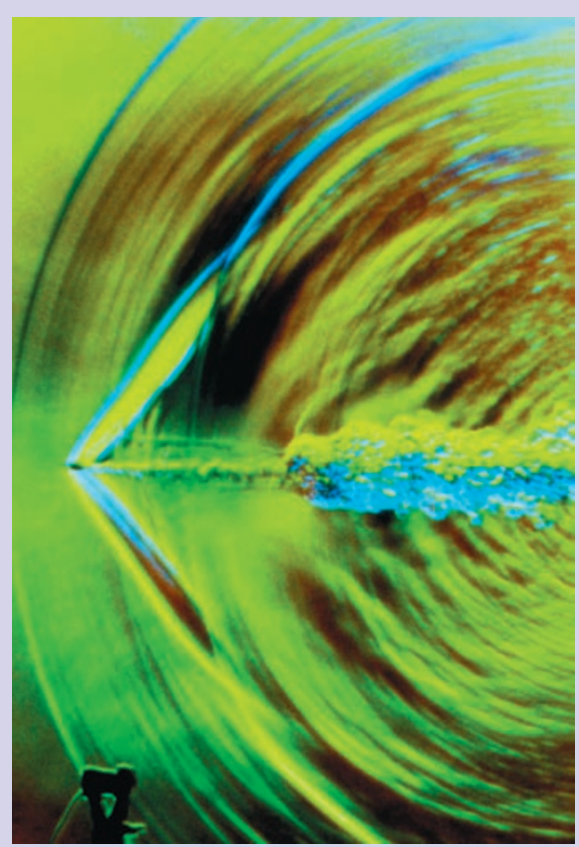

form of the 'minimal supersymmetric standard model', or MSSM. Working with certain constraints to tie the model in with grand unified theory, and in the case that the lightest supersymmetric particle is the gravitino (partner of the graviton), the parameter space of the MSSM extends to negative masssquared values.

This, say Ellis et al., should not be discounted. The point is that any tachyonic spin-zero fields that might exist do so at very high energy scales; in evolving down to the much lower energy scales of our present Universe - a trick that theorists perform using the mathematics of the 'renormalization group' - the sign of the tachyonic masssquared can change, if the actual size of the mass-squared was sufficiently small up at the unification scale.

Models like this do, however, raise other issues: for example, whether our Universe exists in a true minimum of potential or only a local minimum, and whether there is a danger of introducing charge and colour-charge non-conservation. But these, the authors stress, are points that can be reasonably considered (or calculated) without wantonly discarding the tachyon.

Alison Wright 